BULL. AUSTRAL. MATH. SOC.

VOL. $10(1974), 1-8$.

\title{
Four-fold torsion theories
}

\section{Edgar A. Rutter, Jr}

In this note 4-fold torsion theories (for categories of modules) are classified by means of orthogonal pairs of comaximal ideals. Among the applications are results of Kurata concerning lengths of $n$-fold torsion theories and an upper bound for the number of 4-fold torsion theories over a semiperfect ring.

\section{Introduction}

Let $R$ denote a ring with identity. Kurata [2] has introduced the notion of an $n$-fold torsion theory for the category $R$-mod of left $R$-modules. For an integer $n>1$, this is an $n$-tuple

$$
\left(T_{1}, \ldots, T_{n}\right)
$$

of classes of $R$-modules such that each successive pair $\left(T_{i}, T_{i+1}\right)$, for $i=1, \ldots, n-1$, forms a torsion theory. The $T_{i}$-torsion submodule of an $R$-module $M$ is denoted by $t_{i}(M)$. (For an excellent account of torsion theories and their relation to topologies and radicals see Chapter 1 of Stenström [5].)

If there exists an integer $I<i<n$ such that $T_{1}=T_{i+1}$, then the smallest such integer is the length of $\left(T_{1}, \ldots, T_{n}\right)$. If not, $\left(T_{1}, \ldots, T_{n}\right)$ has length $n$.

Kurata [2] proved that there are essentially only four types of $n$-fold torsion theories:

(1) 2-fold torsion theories which cannot be extended to 3-fold

Received 19 July 1973. 


\footnotetext{
torsion theories;

(2) 3-fold torsion theories with length 2 ;

(3) 3-fold torsion theories which cannot be extended to 4-fold torsion theories; and

(4) 4-fold torsion theories of length 4 .
}

Furthermore, for commutative rings and semiprime rings only the first three types exist. (Terminology used here without definition can be found in [3] or [5].)

A 3-fold torsion theory is nothing but a TTF-theory defined by Jans [1]. It was shown in [1] that there exists a one-to-one correspondence between TTF-theories and idempotent ideals of $R$. Furthermore, the TTF-theories of length 2 are precisely those which correspond to ideals generated by a central idempotent. The purpose of this note is to apply the correspondence in [1] to obtain a one-to-one correspondence between 4-fold torsion theories and orthogonal pairs of comaximal ideals of $R$ or, equivalently, ideals of $R$ which are generated as a one sided ideal by an idempotent element. It is possible to determine from such a pair of ideals the length of the corresponding 4-fold torsion theory. This correspondence is used to recover and extend slightly the results of Kurata mentioned previously. New results obtained from this correspondence include a one-to-one length preserving correspondence between 4-fold torsion theories for $R$-mod and those for $\bmod -R$ and an upper bound for the number of 4-fold torsion theories over a semiperfect ring.

\section{The correspondence and applications}

Since the correspondence in Jans [1] is crucial to all that follows, we begin this section by describing it. An idempotent ideal $I$ of $R$ determines three classes of left $R$-modules:

$$
\begin{aligned}
& C_{I}=\{M \text { in } R-\bmod \mid I M=M\} ; \\
& T_{I}=\{M \text { in } R-\bmod \mid I M=0\} ;
\end{aligned}
$$

and

$$
F_{I}=\{M \text { in } R-\bmod \mid\{x \in M \mid I x=0\}=0\} \text {. }
$$


THEOREM A. There exist inverse one-to-one correspondences between 3-fold torsion theories for $R$-mod and idempotent ideals of $R$ given by $\left(T_{1}, T_{2}, T_{3}\right) \rightarrow t_{1}(R)$ and $I \rightarrow\left(C_{I}, T_{I}, F_{I}\right)$. Furthermore, $\left(T_{1}, T_{2}, T_{3}\right)$ has length 2 if and only if $R=t_{1}(R) \oplus t_{2}(R)$ (ring direct sum).

The information in Theorem A is a summary of Theorem 2.1, Corollary 2.2 and part of Theorem 2.4 of [1].

If $(I, K)$ is a pair of ideals of $R$, it is an orthogonal pair if $I K=0$ and the ideals are comaximal if $I+K=R$.

THEOREM B. There exist one-to-one correspondences between each pair of the following:

(1) 4-fold torsion theories for R-mod;

(2) orthogonal pairs of comaximal ideals of $R$;

(3) ideals $I$ of $R$ such that $I=R e$ with $e^{2}=e \in R$; and

(4) ideals $K$ of $R$ such that $k=f R$ with $f^{2}=f \in R$.

Proof. First we exhibit the correspondence between (1) and (2). Let $\left(T_{1}, T_{2}, T_{3}, T_{4}\right)$ denote a 4-fold torsion theory. Since both $t_{2}(R)$ and $R / t_{1}(R)$ belong to $T_{2}$, it follows from Theorem A applied to $\left(T_{1}, T_{2}, T_{3}\right)$ and $\left(T_{2}, T_{3}, T_{4}\right)$, respectively, that $t_{1}(R) t_{2}(R)=0$ and $t_{2}(R)\left(R / t_{1}(R)\right)=R / t_{1}(R)$.

The second equality implies that $R=t_{1}(R)+t_{2}(R)$. Therefore, let

$$
\left(T_{1}, T_{2}, T_{3}, T_{4}\right) \rightarrow\left(t_{1}(R), t_{2}(R)\right)
$$

If $(I, K)$ is an orthogonal pair of comaximal ideals, it is clear that $I$ and $K$ are idempotent. Also for any $R$-module $M, I M=0$ if and only if $K M=M$. Thus $T_{I}=C_{K}$. Similarly, $F_{I}=T_{K}$. It follows from Theorem A that $\left(C_{I}, T_{I}, F_{I}, F_{K}\right)$ is a 4-fold torsion theory and that the correspondence

$$
(I, K) \rightarrow\left(C_{I}, T_{I}, F_{I}, F_{K}\right)
$$

and the correspondence given above are inverses. 
lext we exhibit the correspondence between (2) and (3).

If $(I, K)$ is an orthogonal pair of comaximal ideals, $1=e+f^{\circ}$ with $e \in I$ and $f \in K$. It is immediate from the orthogonality of $I$ and $K$ that $e$ and $f$ are orthogonal idempotents with $I=R e$ and $K=f R$. Let

$$
(I, K) \rightarrow I=R e
$$

If $I=R e$ with $e^{2}=e$ is an ideal, so is $K=(1-e) R$ and $I+K \supset e R+(1-e) R=R$. Let

$$
R e \rightarrow(R e,(1-e) R) .
$$

If $(I, K)$ and $(I, L)$ are orthogonal pairs of comaximal ideals, $K=(I+L) K=I K+L K=L K \subset L$. Similarly $L \subset K$ and so $K=L$. Thus the above correspondences are inverses.

By symmetry there exists a one-to-one correspondence between (2) and (4). The balance of the proof follows by composing the correspondences now in hand.

COROLLARY 1. Every 4-fold torsion theory has length 2 or 4. Moreover, $\left(T_{1}, T_{2}, T_{3}, T_{4}\right)$ has length 2 if and only if $t_{2}(R) t_{1}(R)=0$.

Proof. Let $I=t_{1}(R)$ and $K=t_{2}(R)$. We first prove the second assertion. By Theorem $A,\left(T_{1}, T_{2}, T_{3}, T_{4}\right)$ has length 2 if and only if $R=I \oplus K$. By Theorem B, $R=I+K$ and $I K=0$. Thus $R=I \oplus K$ if and only if $I \cap K=0$. But

$$
K I \subset I \cap K=(I+K)(I \cap K)=I(I \cap K)+K(I \cap K) \subset I K+K I=K I .
$$

Now we establish the first assertion. If $\left(T_{1}, T_{2}, T_{3}, T_{4}\right)$ does not have length $2, I K \neq 0$. By Theorem $B, K=f R$ with $f^{2}=f$, so $K I \in F_{K}=T_{4}$. But $I(K I)=0$, so $K I \notin C_{I}=T_{1}$. Thus $T_{1} \neq T_{4}$, and $\left(T_{1}, T_{2}, T_{3}, T_{4}\right)$ has length 4 .

The first part of Corollary 1 is due to Kurata [2, Proposition 3.2].

EXAMPLE. If $R$ is a left artinian ring with zero left singular 
ideal, it is readily verified that the left socle of $R$ is an ideal which is faithful as a right ideal and has the form $f^{2} R$ with $f^{2}=f \in R$. For rings with zero left singular ideal, the Goldie torsion theory and the dense torsion theory coincide. (See [5].) In the present circumstances, they are equal to $\left(T_{f R}, F_{f R}\right)$. It is immediate from Theorem B that this torsion theory can be extended to a 4-fold torsion theory. If $R$ is not semisimple, so that $f R \neq R$, it follows from Corollary 1 that the resulting 4-fold torsion theory has length 4 .

Numerous other examples of 4-fold torsion theories of length 4 may be obtained by applying the next corollary in conjunction with Corollary 1. A natural source of examples is rings of triangular matrices over, for instance, a division ring.

Let $R$ be a semiperfect ring and $E=\left\{e_{1}, \ldots, e_{n}\right\}$ be a complete set of primitive orthogonal idempotents of $R$. A subset $S$ of $E$ is triangular, provided $e R(1-e)=0$ where $e=\sum e_{i}$ such that $e_{i} \in S$.

COROLLARY 2. Let $R$ be a semiperfect ring and $E$ be a complete set of primitive orthogonal idempotents of $R$. There exists a one-to-one correspondence between 4-fold torsion theories for $R$-mod and trianguzar subsets of $E$.

Proof. In view of Theorem B, it suffices to exhibit a one-to-one correspondence between triangular subsets $S$ of $E$ and orthogonal pairs of comaximal ideals of $R$. Let $e=\sum e_{i}$ such that $e_{i} \in S$. Clearly $R e$ is an ideal. Thus we can let

$$
S \rightarrow(R e,(1-e) R)
$$

To obtain the inverse correspondence we first prove that if $(I, K)$ is an orthogonal pair of comaximal ideals of $R$, then for each $e_{i} \in E$ either

$$
I R e_{i}=R e_{i} \text { and } e_{i} R K=0
$$

or

$$
I R e_{i}=0 \text { and } e_{i} R K=e_{i} R
$$


Clearly $R e_{i}=(I+K) R e_{i}=I R e_{i}+K R e_{i}$. Thus $I R e_{i} \neq J e_{i}$ or $K R e_{i} \notin J e_{i}$, where $J$ is the Jacobson radical of $R$, since $J e_{i}$ is the unique maximal submodule of $R e_{i}$. hence $R e_{i}=I R e_{i}$ or $R e_{i}=K R e_{i}$. In the first instance, $\quad e_{i} R \subset I$ and so $e_{i} R K=0$. Now suppose $R e_{i}=K R e_{i}$. Clearly $I R e_{i}=0$. By symmetry, $e_{i} R=e_{i} R K$ or $e_{i} R=e_{i} R I$. The latter equality would contradict the fact that $I R e_{i}=0$. Thus we have established the above dichotomy. It is immediate from this observation that $I=R e$ and $K=(1-e) R$ where $e=\sum e_{i}$ such that $e_{i} \in E$ and $I R e_{i}=R e_{i}$. It can now be readily verified that the correspondence

$$
(I, K) \rightarrow S=\left\{e_{i} \in E \mid I R e_{i}=R e_{i}\right\}
$$

is the inverse of the one described previously.

The preceding corollary implies that for a semiperfect ring there exist only finitely many 4-fold torsion theories. We sharpen this observation somewhat.

COROLLARY 3. Let $R$ be a semiperfect ring with $k$ isomorphism types of primitive idempotents. There exist at most $2^{k}$ 4-fold torsion theories for $R$-mod, not more than $2^{k-1}-1$ of which have length 4 .

Proof. Let $E$ be a complete set of primitive orthogonal idempotents for $R$. The elements of $E$ can be indexed as $e_{i j}$, with $i=1, \ldots, k$ and $j=1, \ldots, q_{i}$, so that $R e_{i j}$ is isomorphic to $R e_{u v}$ if and only if $i=u$. If $S$ is a triangular subset of $E$ and $e_{i m} \in S$ then $e_{i j} \in S$ for each $j=1, \ldots, q_{i}$. Thus distinct triangular subsets of $E$ determine distinct subsets of $\{1, \ldots, k\}$. The first assertion now follows from the preceding corollary. The empty set and $E$ are vacuously triangular subsets of $E$ but the corresponding 4-fold torsion theories have length 2 . Thus the possibilities in the second instance are reduced to $2^{k}-2$. However, if $S \neq E$ is a non-empty subset of $E$ with both $S$ and its complement triangular, it is immediate from Corollary 1 that the 4-fold torsion theory associated with $S$ has length 2 . Thus 
there are at most $\left(2^{k}-2\right) / 2=2^{k-1}-14$-fold torsion theories of length 4 .

COROLLARY 4 (Kurata). If $n>4$, any $n$-fold torsion theory has length 2 .

Proof. Let $I=t_{1}(R), K=t_{2}(R)$ and $L=t_{3}(R)$. By Theorem $\mathrm{B}$, $(I, K)$ and $(K, L)$ are orthogonal pairs of comaximal ideals of $R$. Thus $I=I(K+L)=I K+I L=I L \subset L$. Similarly, $L \subset I$ and so $L=I$. Hence $I K=0$ and the conclusion follows from Corollary 1 .

By the uniqueness of the torsion theory corresponding to any torsion class for $R-\bmod$ (see [5] or [1]), it is clear that for any integer $n \geq 3$ an $n$-fold torsion theory of length 2 is obtained by alternate repetition of the first two classes. Thus the next corollary is a consequence of Corollaries 1 and 4.

COROLLARY 5 (Kurata). There exist only four different types of n-fold torsion theories:

(1) 2-fold torsion theories which cannot be extended to 3-fold torsion theories;

(2) 3-fold torsion theories of length 2 ;

(3) 3-fold torsion theories which cannot be extended to 4-fold torsion theories; and

(4) 4-fold torsion theories of length 4.

The next result also stems from Corollaries 1 and 4 .

COROLLARY 6. For every integer $n>3$, alz n-fold torsion theories for $R$-mod have length 2 if and only if for every orthogonal pair $(I, K)$ of comaximal ideals of $R$ the pair $(K, I)$ is orthogonal.

COROLLARY 7. Each of the following classes of rings has the property that for every integer $n>3$ all $n$-fold torsion theories have length 2 :

(1) commutative rings;

(2) semiprime rings; and

(3) left or right injective cogenerator rings. 
Proof. This is an application of the preceding corollary. It clearly applias in the first two instances and the third case follows from [4, Theorem 7].

The first two parts of this corollary are due to Kurata [2, Propositions 4.4 and 4.6$]$.

The final corollary is immediate from Theorem B, Corollary 1, and their right hand analogs.

COROLLARY 8. There exists a one-to-one correspondence between 4-fold torsion theories for $R$-mod and 4-fold torsion theories for mod- $R$ which preserves length.

\section{References}

[1] J.P. Jans, "Some aspects of torsion", Pacific J. Math. 15 (1965), 1249-1259.

[2] Yoshlki Kurata, "On an n-fold torsion theory in the category $R^{M}$ ", J. AZgebra 22 (1972), 559-572.

[3] Joachim Lambek, Lectures on rings and modules (Blaisdell, Waltham, Massachusetts; London; Toronto; 1966).

[4] Edgar A. Rutter, Jr, "Torsion theories over semiperfect rings", Proc. Amer. Math. Soc. 34 (1972), 389-395.

[5] Bo Stenström, Rings and modules of quotients (Lecture Notes in Mathematics, 237. Springer-Verlag, Berlin, Heidelberg, New York, 1971).

Department of Mathematics,

Virginia Polytechnic Institute and State University,

Blacksburg,

Virginia,

USA. 